\title{
Analysis of the Effect of Enriched Psychological Nursing Intervention for Patients Who Undergo Family Planning Operations
}

\author{
Xingxiu $\mathbf{L i}^{*}$ \\ Family Planning Service Station of Shangri-La County, Diqing, Yunnan 674400, China
}

\begin{abstract}
Objective: To explore the effect of enriched psychological nursing intervention in family planning operations, to provide a reference for the nursing work. Method: The 90 patients who were treated in our hospital from January 2014 to October 2014 were randomly divided into two groups. The control group received routine nursing care and the experimental group received enriched psychological nursing intervention. The anxiety, pain and complications of the two groups were compared, and the Results were statistically analyzed. Result: The patients in the experimental group were treated with psychological nursing intervention. After the surgery, the state of nervous anxiety was significantly lower, the postoperative pain was mild and the complications were less. When compared with the control group, there were significant differences $(p$ $<0.05)$. Conclusion: The enriched psychological nursing of patients in family planning operations can help to improve the quality of nursing and appease the mood of the patients with a positive clinical significance.
\end{abstract}

\section{KEYWORDS}

Family planning operation

Psychological nursing

Clinical effect

Artificial abortion

\section{Introduction}

Family planning operations are mainly undergone by healthy women of childbearing age. These operations include artificial abortions, induced abortion, normal tubal ligation, intrauterine device (IUD) placement operation, etc. Those who undergo such procedures may experience post-operative uterine perforation, bleeding, endocrine dysfunction, irregular menstruation and so on. In recent years, more and more artificial abortion operations are done in various hospitals, mainly due to, the opening of western hospitals in our country. Artificial abortion is a more commonly used remedial measure after contraceptive failure. Statistical data from the last few years has led to the discovery of the fact that artificial abortion in our

Copyright (C) 2016 Xingxiu Li

doi: $10.18686 /$ jn.v5i2.17

Received: June 26, 2016; Accepted: August 10, 2016; Published online: August 29, 2016

This is an open-access article distributed under the terms of the Creative Commons Attribution Unported License (http://creativecommons.org/ licenses/by-nc/4.0/), which permits unrestricted use, distribution, and reproduction in any medium, provided the original work is properly cited.

${ }^{\star}$ Corresponding author: Family Planning Service Station of Shangri-La County, Diqing, Yunnan 674400, China. E-mail: lixingxiu123y@sina.com country tends to be done for younger patients. There is lack of awareness about contraceptive techniques amongst the younger generation.

Drawbacks of contraception: Patients who underwent abortion are suffered with serious mental stress problems. Complications of artificial abortion usually appear after the surgery [1]. Not only that, patients who undergo family planning surgeries are often affected by a series of psychological problems due to personal, social and family factors. To solve the mentioned problems, our service station has enriched the intervention of the psychological nursing in artificial abortions, and achieved good results.

\section{Materials and methods}

\subsection{The general information}

The 90 patients who were treated in our hospital from January 2014 to October 2014 were randomly divided into two groups. All of the patients underwent artificial abortion voluntarily, after the $\mathrm{B}$ ultrasound examination was found to be normal.

There were 45 patients in the control group, ages between 18 and the 42 with the average of $(25.24 \pm 4.87)$ years old. There were 24 married persons and 21 cases of unmarried. There were 17 cases of non-contraception and 
28 cases of contraceptive failure. The menopause duration is $40-77$ days, the average being $(51.48 \pm 6.39)$ days. In the experimental group, 45 patients were operated, ages between 45 and 19 with an average of $(25.54 \pm 5.71)$ years. There were 25 married patients and 20 unmarried patients. Besides, 19 cases are without contraception and 26 cases have failed in contraception. The menopause duration is 42 to 78 days, the average being $(51.96 \pm 5.07)$ days. There was no significant difference between the two groups regarding the age, marital history and menopause, $p>0.05$, so they were comparable.

\subsection{Method}

The control group received routine nursing care and the experimental group received enhanced psychological nursing intervention. The anxiety, pain and complications of the two groups were compared. The degree of satisfaction of the nursing care was evaluated by the patient.

\subsection{The intervention psychological nursing}

During surgery, nurses should give kind nursing care with enthusiasm to create relax and harmonious environment in order to reduce psychological burden of patient. They should also be patient and careful to explain the methods and advantages of the artificial abortion procedure, to help the patients establish confidence. The nurses should provide guidance and clear explanations to the patients who have severe anxiety. If the patients have doubts, the nurses should be patient to answer clearly and articulately. They should be ready to communicate in either language to avoid the patients' suspicion [2]. Nurses must work with an alert mind, so that the patient can establish their sense of security.

After the surgery, the nurse should actively communicate with the subject. They should help the patients to keep their minds off the surgery and divert their attention. The nurse can hold patients' hands and wipe sweat off their face. The nurse can also press the acupoints to relieve their pain. The nurse should provide warm water, help them to clean up the vomiting objects, comfort and encourage the patients. Before subjecting the patient to the psychological nursing intervention, the nurses should understand the whole situation, including demographics and take individual differences into account. As for patients with low edu- cational level, the nurses not only have to provide psychological comfort, but also pay attention to meet the needs of the patients in terms of contraception awareness. For busy patients, nurses should inform them of the importance of rest after the operation.

\subsection{Observation index}

After the surgery, the patient's anxiety, pain, complications and satisfaction were measured in the form of a questionnaire. In the process of filling the questionnaire, the patients and their families were explained about its contents in detail. The patients were asked to fill the questionnaire in real time to ensure the accuracy of the study.

\subsection{The data processing}

The experimental data were recorded in the SPSS18.0 software package and analyzed statistically. For $95 \%$ of the confidence interval, $p<0.05$, the difference was statistically significant. The mean + standard difference $(\chi \pm s)$ was determined for the state of pain and anxiety. The groups were compared using $t$ test; complication and satisfaction were expressed as the number of cases $(n)$ and percentage (\%); and the results were compared using $\chi^{2}$ test.

\section{Results}

After the psychological nursing intervention, the state of nervous anxiety of the patients in the experimental group was significantly lower than that after the surgery. The postoperative pain was mild and the complications were significantly lower than those of control. The difference was statistically significant $(p<0.05)$. The detailed results are shown in the Table 1.

\section{Discussion}

Family planning surgery is for healthy women at a childbearing age with reproductive concerns. After giving birth, the method of birth control is used to effectively control fertility. A number of women of childbearing age resort to family planning surgeries due to faulty birth control methods, damaged condoms and other reasons that lead to contraceptive failure. Family planning surgery responds to the policy of the national family planning and lowers the family expenses. This surgical procedure is not done to cure any type of woman disease. Therefore, special atten-

Table 1. The comparison of the state of anxiety, pain, complications and satisfaction of the two groups of patients after nursing intervention.

\begin{tabular}{cccccc} 
Group & Case & The anxiety state & Pain & $\begin{array}{c}\text { Complications } \\
\mathrm{n}(\%)\end{array}$ & $\begin{array}{c}\text { Satisfaction } \\
\mathrm{n}(\%)\end{array}$ \\
\hline The experimental group & 45 & $41.85 \pm 7.64$ & $2.08 \pm 0.24$ & $1(2.22)$ & $45(100)$ \\
The control group & 45 & $49.37 \pm 8.89$ & $2.93 \pm 0.52$ & $6(13.33)$ & $38(84.44)$ \\
\hline
\end{tabular}

Note: The state of anxiety of the experimental group is lower than that of the control group, $(t=12.63, p<0.05)$. The pain of the experimental group is lower than that of the control group, $(t=8.26, p<0.05)$. The complications of the experimental group are lower than that of the control group, $\chi^{2}=5.34, p<0.05$; the satisfaction of the experimental group is higher than that of the control group, $\chi^{2}=6.94$, $p<0.05$. 
tion should be given to the psychological state of patients undergoing this type of surgery. The patients are generally lack of knowledge about the family planning operation due to their cultural level is relatively low. They often show tension, anxiety and fear before the operation which resulting in psychological and emotional instability, and affecting the postoperative recovery. At the same time, artificial abortion can be considered to be traumatic to the woman as it greatly influences the body and mind. An effective measure of nursing intervention is a key to promote the rehabilitation of patients, reduce the rate of recurrent abortion and improve the effectiveness of contraception [3].

The clinical nursing work in our service station has enhanced the psychological nursing intervention and appeased the emotion of patient. Nurses can understand the psychological state of the patients and provide them with targeted nursing techniques [4] through communication with the patient. For example, the nurse should inform the patients who are more psychologically affected about the advantages of the artificial abortion, like the post-surgery damage to the uterus is small and it will not affect subsequent births, etc. Some young unmarried women did not use contraceptive measures, they are over-trusted to abortion and even underwent artificial abortion repeatedly. The nurse should inform them that the artificial abortion has a certain harm and the contraceptive measures are the keys to ensure their reproductive health $[5,6]$. In order to do nursing work effectively, nursing staff should start from the basics. In the process of implementing nursing work, to aim for people-oriented techniques, enhance the awareness of patients, respect patients, and build rapport with patients, to solve misunderstandings between doctors and patients and to lay the foundation for a better, more positive view of nursing staff. After the use of psychological care, the state of anxiety of the patients in the experimental group is reduced, the pain is relieved and the complications are reduced. Compared with the control group, there was significant difference, $p<0.05$. The results of this study show that the two groups of patients after the implementation of the family planning surgery, using a different approach. The observation group adopted the method of nursing intervention, and achieved some results. In addition to the above results, the total degree of satisfaction is good. Among them, the total satisfaction of the observation group was $100 \%$, while the control group's total satisfaction was $84.44 \%$.The observation group was signifi- cantly higher than the control group. The total satisfaction of the two groups was significantly different $(p<0.05)$. Further explanation in the family planning operation has a positive clinical significance. It helps to enrich the psychological nursing of the patients, improve the quality of nursing, and to appease the emotional of patients $[7,8]$.

In summary, family planning is a long-term and arduous work. In the process of carrying out family planning surgery, the patient's psychological problem has been concerned. After the patient received the plan of parenthood, nursing staff should always pay attention to patients' psychological change and psychological mood. The nurse should give patients appropriate guidance and psychological comfort to the patients. Effective nursing care helps to avoid the adverse psychological mood of the patients which will affect the postoperative recovery. Therefore, enriched psychological care of patients for family planning surgery has a positive clinical significance as it can help to improve the quality of care and effectively appease the patient's mood.

\section{References}

1. Liang X. Psychological Analysis and Psychological Nursing on Patients with Family Planning. International Journal of Pharmaceutical Medicine. 2006;12(3):67-68.

2. Li SM, Feng XH. The Experience of The Psychological Nursing of the Family Planning. Medical Information. 2010;23(8):182.

3. Xia S and Ding S. On the Important Role of Psychological Nursing in Family Planning Surgery. Modern Health Care and Medical Research. 2007;4(26):127.

4. Pan Y. Analysis of the Quality of Care in Family Planning Counseling. Guangzhou Medicine. 2008;39(3):67-68.

5. Yue $\mathrm{CH}$, Wang $\mathrm{X}$, Man $\mathrm{C}$. The analysis of the family Planning Surgery in Patients with the Psychological Problems and the Nursing Intervention Measures. The Chinese Medicine. 2014;2(6):957-959.

6. Li S, Zou Y, Chu G. Family Planning Surgery and Women's Mental Health. Chinese Journal of Family Planning. 2012;1(7):499-501.

7. Tan D. Research on The Prevention And Control Strategies Of The Complication Of Family Planning Operation And Prevention And Control. Journal of Nursing Practice and Research. 2013;6(19):69-70.

8. Lu M, Xu X. The Application of the Psychological Nursing Intervention in the Treatment of Female Tubal Ligation. Modern Medicine. 2015;8(3):239-240. 